\title{
AIREACIÓN EN LA TECNOLOGÍA BIOFLOC (BFT): PRINCIPIOS BÁSICOS, APLICACIONES Y PERSPECTIVAS
}

\author{
Javier Piñeros-Roldan1, Mariana Catalina Gutiérrez-Espinosa², Mauricio Gustavo Coelho- \\ Emerenciano $^{3}$, Marina Lapa-Viana ${ }^{4}$
}

${ }^{1}$ MVZ. Esp. cMSC Acuicultura Universidad de los Llanos, ${ }^{2} \mathrm{CDra}$ Ciencias Agracias Universidad de los Llanos, ${ }^{3}$ Santa Catarina State University (UDESC), ${ }^{4}$ Asian Institute of Technology (AIT). Correo de correspondencia: javiermvz@outlook.com

\begin{abstract}
RESUMEN
En un mundo globalizado de creciente población ávido por nutrientes para su alimentación, la proteína de origen acuática emerge como la gran alternativa nutricional del siglo. La disminución de sus recursos para la explotación (agua, tierra y personal) implica que se deba intensificar la producción de peces y camarones y una posibilidad para dicho establecimiento es la tecnología de biofloc (BFT en su sigla en inglés). Sin embargo, esta intensificación debe ser dada con principios de eficiencia técnica y económica, por lo tanto, un correcto diseño de la aeración es de fundamental importancia para mantener las partículas en suspensión, oxigenar y mover correctamente el agua. Unido a los alimentos concentrados, el costo energético (incluyendo la aeración) son de los más importantes costos de producción usados en la tecnología BFT. Utilizando recambios mínimos o nulos, el número, tipo y distribución de los aparatos mecánicos de aeración son cruciales para el mantenimiento de la calidad de agua para las especies cultivadas y para los microorganismos que se encuentran inmiscuidos en el sistema. En este contexto, esta revisión pretende brindar una base teórica para el estudio e implementación de los sistemas de aireación en los sistemas BFT, además de trazar perspectivas futuras para estos componentes.
\end{abstract}

Palabras Clave: Biofloc; densidades de siembra; cultivo intensive; peces; camarones; acuacultura; biomasa; tasa de consumo de oxígeno.

Recibido: 20 de Septiembre de 2019. Aceptado: 11 de Mayo de 2020

Received: September 20, 2019. Accepted: May 11, 2020

\section{AERATION IN BIOFLOC TECHNOLOGY (BFT): BASICS PRINCIPLES, APPLICATIONS AND} PERSPECTIVES

\begin{abstract}
In a globalized world with growing population avid for nutrients for their feeding, the aquatic originated protein emerges as the big nutritional alternative of the century. The reduction of their resources to the farming (water, field and human resource) implies that the fish and shrimp production has to be intensify and one of the possibilities for this development is Biofloc Technology (BFT). Nevertheless, this intensification has to be given with principles of economic and technical efficiency, therefore, a correct design of the aeration is a big principal to maintain the suspended particles, oxygenate and move the water correctly. The electricity (aeration included) join with the feed are the most important production costs using BFT. With water changes low or none of it, the number, type and distribution of the mechanical oxygenator devices are crucial to maintain the water quality for the cultivated species and for the microorganisms that are included on the system. In this context this revision pretends to present the basis for the study and implementation of the aeration systems on BFT, also drawing future perspectives for these components.
\end{abstract}

Keywords: Biofloc; stocking density; intensive farming; fish; shrimp; aquaculture; biomass; oxygen consumption rate.

Cómo citar este artículo: J. Piñeros, M. Gutiérrez, M. Coelho, M. Lapa. "Aireación en la tecnología Biofloc (BFT): principios básicos, aplicaciones y perspectivas", Revista Politécnica, vol.16, no.31 pp.29-40, 2020. DOI: 10.33571/rpolitec.v16n3 


\section{INTRODUCCIÓN}

La pesca y la acuicultura continúan siendo importante fuentes de alimento, ingresos y medios de vida para cientos de millones de personas en todo el mundo (1). La producción de animales acuáticos ha dejado de basarse principalmente en la captura del medio natural para comprender la cría de un número creciente de especies cultivadas. En 2016, la producción acuícola mundial ascendió a 80,03 millones de toneladas, mientras que la pesca de captura obtuvo 90,91 millones de toneladas. De estas 151,2 millones fueron usadas para consumo humano (2) . Sin embargo, este crecimiento se afronta en escenarios alrededor del globo en donde el agua es escasa y la tierra es costosa, por lo tanto se hacen necesarias formas de acuicultura más intensiva donde debe hacerse prioritario producir más en menos espacio, pero sin dejar de lado los aspectos económicos y ambientales (3). Es decir, la producción debe ser económicamente viable y ambientalmente amigable para atender la demanda cada vez más creciente de productos acuícolas, sobre todo de productos considerados "ecológicamente más correctos".

En el sentido de una producción más amigable, fueron desarrollados diversos sistemas de cultivo que son clasificados según su grado de intensificación (4). Los sistemas de producción tradicionales, los más utilizados en todo el mundo, tienen como principal característica bajas biomasas producidas por unidad de área, pero sobre todo, la necesidad de constantes renovaciones de agua para el mantenimiento de su calidad (5). Es aquí donde se presenta el mayor problema debido a que puede producir, por mal manejo, una rápida acumulación de residuos diversos, materia orgánica y compuestos inorgánicos tóxicos en los ambientes adyacentes (6). Además de eso, los recambios de agua en una misma zona donde están ubicadas diversas granjas acuícolas pueden ayudar a diseminar las enfermedades a una velocidad estruendosa. Desafortunadamente este escenario ya fue visto varias veces alrededor del globo en los cultivos del camarón marino con el virus de la mancha blanca (WSSV en su sigla en inglés) (7) y por el síndrome de la mortalidad temprana (EMS) causada por bacterias (8); y más recientemente también con tilapias con el virus del TilV como lo ocurrido en países como Egipto y Colombia (9). Por otro lado, sistemas intensivos, que manejan altas biomasas por unidad de área y una reducida o nula tasa de recambio, son sistemas considerados más controlados y bioseguros, contraponiendo los problemas citados (3). Por lo tanto, para un crecimiento sostenible de la actividad los sistemas actuales deben ser repensados. Desde este punto de vista, el sistema de producción tecnología biofloc (o BFT en su sigla en inglés) es intensivo y considerado una revolución para la acuacultura mundial (10). Con manejo in situ de aguas, este sistema ha sido desarrollado para mejorar el control ambiental de los cultivos (11), proveer alimento suplementario en forma de agregados microbianos y competir con patógenos $(12 ; 13)$.

El término "biofloc" se refiere a un sistema o tecnología de producción realizados en tanques o estanques altamente oxigenados y en constante movimiento del agua (13). Además, las unidades de producción son provistas con altas densidades de siembra y fertilizados con fuentes ricas en carbono para estimular el surgimiento de una microbiota específica (14), tales como bacterias, algas, protozoos, partículas de origen orgánico como heces y alimento no ingerido, que se mantiene unido por moco secretado por las bacterias, microorganismos filamentosos o atracción electrostática (3).

La comunidad microbiana, predominantemente de bacterias heterótrofas, son fomentadas por una fertilización basada en una alta relación entre carbono: nitrógeno (15) con el fin de que el sistema contenga altas concentraciones de bacterias consideradas degradadoras de materia orgánica (15) y que posea un mínimo de $5 \mathrm{~mL} \mathrm{~L}^{-1}$ de sólidos sedimentables (11). Estos valores indican, de manera indirecta, que ya ocurrió una sucesión ecológica deseada en que las comunidades de bacterias heterotróficas se encuentran establecidas (11). Teniendo en cuenta que si el carbono y nitrógeno son bien balanceados (relación entre $10 \mathrm{a}$ 20:1) (13) estos son convertidos en biomasa microbiana (16) y pueden ser usados como alimento para ciertas especies, como es el caso de las tilapias (6) y el camarón marino Litopenaeus vannamei (17), dos de los grupos de especies más utilizados en BFT.

Paralelamente y no menos importantes, otros grupos se desenvuelven en conjunto a la biota heterotrófica al encontrar un ambiente propicio: las bacterias nitrificantes (también llamadas quimio autotróficas) (18). Las bacterias nitrificantes pueden representar cerca de $2 / 3$ de la transformación del nitrógeno amoniacal. Este grupo, que poseen un crecimiento más lento y que tardan en colonizar el medio, no degrada la materia orgánica, pero si oxida el amonio 
en nitrito y el nitrito en nitrato, utilizando carbono inorgánico para su metabolismo (3).

En este sentido, tanto para bacterias degradadoras (Bacillus sp., Lactobacillus sp, etc.) como para nitrificantes, la oxigenación es fundamental para sus metabolismos. Sumado a lo anterior, las altas densidades de siembra y la necesidad de la resuspensión de los agregados hacen que los sistemas de aireación sean rigurosamente dimensionados y distribuidos en los sistemas de producción que utilizan este tipo de tecnología.

Siendo así, el objetivo del presente artículo es recopilar la información disponible acerca del uso de diferentes sistemas de aireación, discutir sobre algunas alternativas actuales y reflexionar sobre las perspectivas en este tema en los cultivos adoptando la tecnología BFT.

\section{DISPOSITIVOS DE AIREACIÓN EN BFT}

Sistemas de producción con mínima renovación de agua y que manejan mayores cargas orgánicas acarrean en un redoble de atención en cuanto al tipo más adecuado, la disposición y dimensionamiento de los dispositivos de aireación (19). En la acuicultura tradicional (con menor biomasa por unidad de área), gran parte de la incorporación del oxígeno disuelto (OD) es originado vía fotosíntesis y por el recambio atmosférico (20), frecuentemente sin uso de aparatos mecánicos y cuando sí, utilizados pocas horas del día (21). Sin embargo, para los sistemas BFT es crucial el uso de los aireadores mecánicos encendidos $24 \mathrm{~h}$ al día, los cuales tienen por objetivos: (i) proporcionar el OD para los organismos cultivados en las densidades requeridas y para los microrganismos presentes en la columna de agua - más de $60 \%$ de la demanda de OD puede venir de los microorganismos - (13); (ii) distribuir horizontalmente y verticalmente el OD en la columna de agua; (iii) evitar la estratificación térmica; (iv) resuspender las partículas (bioflocs), evitando las zonas muertas y acúmulo de lodo ("sludge"); (v) dirigir y drenar el lodo para lugares específicos en el estanque. Por lo tanto, los dispositivos de aireación en BFT no están siendo usados tan solo para su uso primario (es decir incorporar el OD) sino a la vez para un conjunto de funciones específicas y bien definidas.

En términos de los principios físicos de incorporación de oxígeno, los dispositivos de aireación pueden clasificarse en dos grupos: grupo 1) Incorporación vía "agua en el aire", son equipos que lanzan el agua para que tenga contacto con el aire atmosférico, donde ocurre una saturación de oxígeno en las gotas lanzadas, estas retornan al cuerpo de agua transfiriendo el oxígeno captado. Además, perturban la superficie del cuerpo de agua, aumentando el cambio atmosférico; el Grupo 2) Incorporación vía "aire en el agua", donde el oxígeno se difunde en el agua al colocar aire atmosférico en forma de burbujas a grandes presiones $(22 ; 21 ; 23)$.

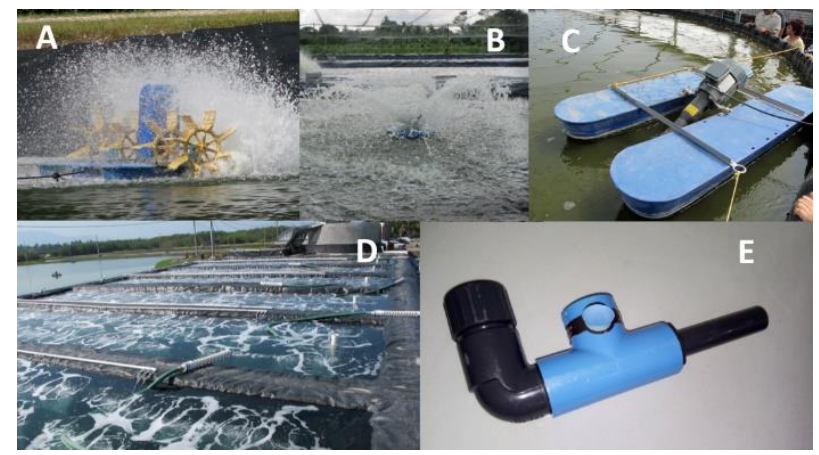

Fig. 1. Principales equipos de aireación utilizados en $\mathrm{BFT}$, de acuerdo con los principios de aireación: grupo 1: Aireadores de paletas y splash (letras A y B); grupo 2: inyectores propulsores, blowers y boquillas inyectoras (venturis) (letras $C, D$ e E). Fotos: Mauricio G. C. Emerenciano.

Según la Figura 1, los principales dispositivos de aireación utilizados actualmente en BFT son los aireadores de paletas y aireadores tipo "splash" (pertenecientes al grupo 1), y también los compresores de aire radiales (también llamados popularmente de "blowers"), los inyectores propulsores y las boquillas inyectoras (venturis), ambos pertenecientes al grupo 2. A continuación se va a discutir las principales características, ventajas y desventajas de cada dispositivo (desde el punto de vista técnico-práctico en observaciones a campo). Vale resaltar que no existe un "mejor equipo", sin embargo, para cada situación pueden existir modelos más adecuados que otros, o hasta un uso mixto con diferentes equipos.

\subsection{Aireador de paletas}

Uno de los principales equipos de aeración utilizados en los cultivos tradicionales de peces y camarones en estanques de tierra. Estos aparatos poseen un sistema de paletas que lanzan el agua del tanque hacia el aire, saturándose. Cuando esta regresa 
incorpora el oxígeno capturado, además de perturbar la superficie del agua, acelerando la transferencia del oxígeno atmosférico y aumentando la concentración de oxígeno disuelto en la columna de agua (21). Este equipo genera una fuerte circulación horizontal/frontal y en menor intensidad un movimiento horizontal/trasero $(24 ; 25)$. El agua lanzada por este dispositivo hace que siempre haya un diferencial hacia la menor concentración de oxígeno, manteniendo constante la captación, oxigenación, difusión y dispersión $(26 ; 25)$. Estos dispositivos son ampliamente empleados en biofloc para cultivo de peces y camarón, este último especialmente en mediana y gran escala. Son también bastante empleados durante ciertos períodos o hasta $24 \mathrm{~h}$ al día (dependiendo de la biomasa presente en el estanque y entrada de alimento concentrado) para facilitar la concentración de los lodos residuales (o "sludge" en su término en inglés) en el centro del estanque para su drenaje.

Ventajas: fácil instalación, generan buen movimiento horizontal/superficial (facilitando la concentración de los lodos) y poseen altos valores de SAE (del inglés "Standard Aerator Efficiency" o Eficiencia Estándar del Aireador).

Desventajas: genera acentuada turbulencia (lo cual puede ser perjudicial para las fases iniciales de cultivo), trabajo unidireccional, pueden perder capacidad de retención térmica en el agua por su modo de operación (en observaciones en campo de los autores fueron detectadas una diferencia de aproximadamente 3 grados comparados con el sistema de compresores radiales), poco movimiento del fondo en estaques más profundos $(>1,2 \mathrm{~m})$.

\subsection{Aireador tipo "splash"}

El modelo tipo "splash" (también llamado tipo fuente o "chafariz") posee distintas conformaciones estructurales responsables por mejores desempeños de unos u otros modelos, como por ejemplo motores de superficie o sumergidos. Estos modelos poseen propiedades de funcionamiento y de diseño que actualmente van siendo ampliamente utilizados con tecnología biofloc, sobre todo en países como Colombia y Brasil (27). En relación con los distintos tipos, según Tucker (54), los modelos con motores de superficie (generalmente utilizados en estanques pequeños y poco profundos) son reconocidos por su eficiencia (buenos valores de SAE). Para su funcionamiento poseen una hélice donde el agua del estanque es lanzada hacia la atmosfera, desencadenando una ruptura de la superficie del agua, transformando una fase líquida homogénea en pequeñas gotas que, al regresar al cuerpo de agua, incorporan moléculas de oxígeno atmosférico. Además, promueven la turbulencia de la superficie acuática oxigenando también por difusión (21). El agua lanzada a la atmosfera va a ser siempre diferente y con una menor cantidad de oxígeno disuelto que la que posee al regresar a la columna de agua, manteniendo así constante la captación, oxigenación, difusión y dispersión $(26 ; 25)$.

Por otro lado los modelos con motores sumergidos poseen el mismo sistema del que tiene motor en superficie, pero el motor trabaja por debajo del nivel del agua funcionando como un contrapeso, de esta manera se aumenta su equilibrio en el centro, reduciendo sus oscilaciones y trepidaciones (28).

Ventajas: trabajo multidireccional, buen rango de circulación de agua vertical (hasta $1.8 \mathrm{~m}$ de profundidad) y eficiencia (SAE) (25). En observaciones de campo de los autores fue detectado una facilidad para concentrar sólidos debajo del dispositivo, fácil manejo e instalación por tratarse de equipos livianos, además de habitualmente presentaren poco mantenimiento.

Desventajas: genera acentuada turbulencia (lo cual puede ser perjudicial para las fases iniciales de cultivo), enfría el agua por su modo de operación y relativamente poco movimiento horizontal/superficial (29).

\subsection{Aireador tipo inyector propulsor.}

Este tipo de aireadores presentan un gran movimiento de agua, puede ser variado su ángulo de inyección y movimiento de agua, lo que en algunos modelos mejora su eficiencia. Poseen por lo general un motor fuera del agua que hace girar un eje que succiona aire del ambiente y lo inyecta en el agua, generando un efecto tipo venturi (29).

Ventajas: en observaciones de campo de los autores fue detectado un buen rango de circulación de agua vertical (alcanzan más de 2,0 m de profundidad), facilidad para generar corrientes y concentrar/dispersar sólidos en puntos específicos, fácil instalación y generalmente presentan poco mantenimiento.

Desventajas: trabajo unidireccional con acentuada turbulencia (caudal muy fuerte), no recomendable a pocas profundidades, pueden promover el rompimiento de los bioflocs (29) y generalmente son menos eficientes (menor SAE; (25)). 


\subsection{Compresores radiales (Blowers)}

Ciertamente es unos de los dispositivos de aireación de mayor uso y difusión dentro de los sistemas BFT a nivel mundial, sobre todo para ambientes más pequeños y controlados (28). En este modelo el sistema se divide básicamente en dos partes, una externa el agua (responsable por la captación del aire atmosférico) y la otra interna del agua (responsable por la dispersión del aire). Vale recordar que aproximadamente $20 \%$ del aire atmosférico es compuesto de oxígeno (30). En la parte exterior del agua los compresores radiales (también llamados de "blowers"), captan el aire por un filtro, pasando por las turbinas y luego es distribuido por los difusores que están dentro del agua, distribuidos en distintos puntos en el fondo. Por lo general, la conducción del aire hasta los difusores es realizada en tubería de policloruro de vinil (PVC), tipo hidrosanitarias y/o de irrigación (esta última generalmente menos costosa). La incorporación de oxígeno en el agua ocurre a través de los distintos tipos de difusores que generan pequeñas burbujas, que, en su tránsito vertical en la columna de agua, dispersa el oxígeno. Cuanto menor el tamaño de las burbujas mayor la relación superficie/área, más lento el tránsito en la columna de agua y consecuentemente mayor la incorporación de oxígeno. Pöpel y Wagner (53) afirman que la disminución del tamaño de las burbujas aumenta su área específica, debido, entre otros, al crecimiento en número de burbujas para ocupar el mismo volumen de agua. En el mercado existen diferentes tipos de difusores con distintos tamaños de burbujas. Antiguamente las propias tuberías de PVC perforadas o las "piedras de aireación" (similares a las de acuarios) eran utilizadas, pero fueron actualmente sustituidas por los discos difusores o por las mangueras micro perforadas, siendo estas últimas por su costo, tamaño de burbujas, vida útil y facilidad de instalación las más utilizadas. Motarjemi y Jameson (9) comentan que burbujas con 2-5 mm de diámetro hacen que la eficiencia de transferencia sea muy baja, por lo que sugieren burbujas de 300 1000 micras de diámetro.

El aire que sale de los difusores generan movimiento en el agua, en general de forma vertical, produciendo cierta corriente que debido a su velocidad constante promueve la circulación del cuerpo de agua y la re-suspensión de las partículas (21). Según Hauser et al. (48), la fuerza de las burbujas generadas debe ser suficiente para generar una turbulencia adecuada; por esta razón distintos dispositivos de control de flujo deben ser instalados en los estanques de producción. En la práctica, la turbulencia adecuada para re-suspender las partículas (biofloc) y no promover demasiado gasto energético en los peces y camarones es de 15-30 $\mathrm{cm} \mathrm{s}^{-1}$ (13). Un punto negativo de la utilización de este sistema se debe a que, por seguridad, contar con más compresores de reserva (backup), pues en una posible falla mecánica del aparato los estanques abastecidos se verán afectados.

Existen distintas marcas y modelos de mangueras micro perforadas en el mercado, variando tanto en términos de calidad de material, presión requerida por metro lineal de manguera en dada profundidad, espesura y tamaño de burbuja generada. Su efectividad se ve directamente relacionada con la profundidad a la cual se introducen al sistema. Es decir, se aumenta la transferencia de oxígeno al disminuir el tamaño de las burbujas, pero también al aumentar la profundidad de dispersión de las mismas (34). En este contexto, es necesario tener en cuenta un diseño adecuado, con el compresor apropiado para el número de difusores, pero también con el metraje de mangueras necesaria de acuerdo con la profundidad y biomasas utilizadas.

Ventajas: buena eficiencia (SAE) utilizando difusores adecuados, mueve de manera eficiente toda la columna de agua (29), generan una turbulencia moderada (lo cual es indicado para fases iniciales de cultivo). En observaciones de campo de los autores fue detectado que la temperatura del agua se mantiene más equilibrada (menor intercambio de calor con el aire atmosférico) y los equipos generalmente requieren poco mantenimiento.

Desventajas: dependiendo del modelo de compresor puede perder eficiencia a mayores profundidades, necesidad de equipos de respaldo (backup) y relativa dificultad de uso para estanques con áreas mayores.

\subsection{Boquillas inyectoras o "nozzles"}

Considerados uno de los sistemas más recientes en BFT (29). Estos sistemas que utilizan bombas de agua, por lo general de tipo centrifuga de alta presión, son basados en una inyección continua de mezclas de gases y agua. Esta inyección es realizada por medio de boquillas (también llamados de "nozzles") que succionan el aire atmosférico por el principio de "venturi" y a través de tubos de 
succión (o "snorkels"). La circulación continua de agua en las boquillas resulta en chorros de pequeñas burbujas que se separan a medida que el flujo se dispersa, ascendiendo en dirección a la superficie del agua. En algunos conjuntos de motobombas y boquillas ("sets") la dimensión de las burbujas es controlada a través del ajuste del vacío o de la presión de operación.

Actualmente en muchos países la mayor dificultad en el uso de estos dispositivos es encontrar el "set" ideal de bomba-boquillas. Para alcanzar una buena tasa de transferencia de oxígeno las boquillas deben trabajar con una presión ajustada dependiendo del fabricante, pero también de la bomba utilizada. Además, los materiales de las bombas deben ser resistentes a agua salada en el caso de cultivos marinos. Dependiendo del modelo existe una limitación cuanto a profundidad de instalación de las boquillas, que a su vez también está relacionada con la tasa de transferencia del oxígeno disuelto $(35 ; 35)$. De acuerdo con Krummenauer et al. (58), los índices de SOTR y SAE de distintos "sets" son totalmente relacionados con el tipo de bombas, su potencia y numero de boquillas.

Ventajas: fondos sin obstáculos (cuando instalados en los laterales), tamaño compacto, eficiencia (SAE) cuando correctamente dimensionado, vida útil prolongada de los materiales con difícil obstrucción de las boquillas (29).

Desventajas: de acuerdo con observaciones a campo hecha por los autores son la dificultad en ajustar un correcto "sef" de motobomba y boquillas dependiendo del país y disponibilidad de equipos, costo, pocos datos productivos disponibles en la literatura, necesidad de mano de obra más específica para el mantenimiento de las bombas y necesidad de sistemas de backup en caso de falla de las mismas.

\subsection{Sistemas mixtos}

Actualmente países como Brasil están evaluando el empleo del uso combinado de dispositivos de aeración en cultivos en BFT (28), con el objetivo de utilizar las mejores ventajas de cada equipo. La combinación de distintos equipos puede promover un mejor movimiento de agua, una mejor transferencia de oxígeno disuelto, además de optimizar el uso energético en los estanques de cultivo, sobretodo en estanques más profundos. En la práctica, muchas combinaciones han sido empleadas tales como aireador de paletas con blowers (13), paletas con aireador tipo splash (observaciones a campo en Colombia), paletas con inyectores propulsores (36), blowers y boquillas inyectoras (28).

\section{FACTORES EN LA SELECCIÓN DEL TIPO DE AIREADOR}

Son innumerables los factores que deben ser tenidos en cuenta antes de escoger un sistema de aireación como su eficiencia, costos del equipo y mantenimiento, la facilidad de instalación, diseño y dimensiones de los estanques, la productividad deseada, entre otros. Sin embargo, para el propósito de esta revisión se trataron de agrupar entre los más importantes.

\subsection{Eficiencia y costo de los equipos}

Para que un sistema funcione y sea eficiente es necesario considerar la cantidad de oxígeno que los aireadores pueden transferir al agua, y esto puede ser dado sobre todo por la Tasa Estándar de Transferencia de Oxígeno (SOTR), y la Eficiencia Estándar del Aireador (SAE) (23). Según este autor, el SOTR (en inglés Standar Oxygen Transfer Rate) es la masa de oxígeno que un aparato puede introducir en un cuerpo de agua por unidad de tiempo a condiciones estándar (temperatura del agua $20^{\circ} \mathrm{C}$, concentración de oxígeno inicial $=0$ $\mathrm{mg} / \mathrm{L}$; una atmosfera de presión y agua clara) medida en $\mathrm{Kg} \mathrm{O}_{2} /$ hora. Ya el SAE (o Standar Aerator Efficiency) es definida como el SOTR por unidad de energía (37), generalmente medida en Kilowatts (Kw) (Tabla 1).

Tabla 1. Ecuaciones y unidades utilizadas para el desarrollo de los valores del SOTR y SAE.

\begin{tabular}{|c|c|c|}
\hline Parámetros & Ecuaciones & Unidad \\
\hline \multirow[t]{2}{*}{ KlaT } & 1,1 & $h-1$ \\
\hline & $\overline{t(70 \%)-t(10 \%)}$ & \\
\hline $\mathrm{KlaT}_{20}$ & KlaT $x 1,023^{(20-T)}$ & $h^{-1}$ \\
\hline SOTR & $K_{20} \times \operatorname{Cs} \times \vee \times 10^{-3}$ & $\underset{\mathrm{h}^{-1}}{\mathrm{Kg} \mathrm{O}_{2}}$ \\
\hline \multirow[t]{2}{*}{ SAE } & SOTR & $\mathrm{Kg} \mathrm{O}_{2}$ \\
\hline & $\overline{\text { Potência motor }(k w)}$ & $(\mathrm{kw} \cdot \mathrm{hr})^{-1}$ \\
\hline
\end{tabular}


$\mathrm{T}=$ Temperatura del agua .

KlaT = Coeficiente de transferencia de oxígeno a la temperatura del agua.

Kla20 = Coeficiente de transferencia del agua a $20^{\circ} \mathrm{C}$.

Cs = Concentración de OD (mg. L-1) a la salinidad probada a $20^{\circ} \mathrm{C}$.

$\mathrm{V}=$ Volumen del tanque de prueba $(\mathrm{L})$

SOTR= Tasa estándar de transferencia de oxígeno.

$\mathrm{SAE}=$ Eficiencia estándar del aireador.

En este sentido, uno de los puntos cruciales de la elección de los equipos es comparar sus precios individuales y fundamentalmente observar los datos de SOTR y SAE lo cual representa su eficiencia. Es conocido que los costos de energía eléctrica son, en promedio, responsable por $30 \%$ de los costos de producción en sistemas BFT (13). Este impacto es sobre todo debido a las exigencias de oxigenación y re-suspensión, necesarias para un buen funcionamiento del sistema. En este sentido, se torna imprescindible que los aireadores utilizados trabajen 24 horas al día durante todo el ciclo de cultivo, lo que justifica la búsqueda de aireadores más eficientes. Liang et al. (45) plantearon estrategias de prender y apagar los aparatos por lapsos de 30 minutos llegando a un ahorro de hasta el $50 \%$ de la electricidad para mantener niveles óptimos para la especie de cultivo.

\subsection{Ambiente de producción (salinidad)}

El ambiente de producción también genera un ítem adicional a la hora de seleccionar los aparatos de aireación, pues no siempre los materiales con los que vienen elaborados permiten el uso en todos los medios del cultivo utilizados en sistemas BFT, tal es el caso de aguas dulces, saladas o salobres. En este sentido, los distintos materiales de construcción de los sistemas mecánicos de los equipos brindan variados grados de durabilidad de acuerdo con el ambiente.

La salinidad y otros factores ambientales presentes en el cultivo se utilizan para los cálculos de aireación en el sistema. Vinatea y Carvalho (32) observaron que a una temperatura de $28^{\circ} \mathrm{C}$ con una salinidad de cero ppt (0) el agua estaba saturada a 7,8 mg/L. Sin embargo, a la misma temperatura, pero a una salinidad de 35 ppt la saturación fue dada con 6,4 $\mathrm{mg} / \mathrm{L}$. Así es importante tener en cuenta la salinidad del ambiente para estimar la tasa de transferencia de los diferentes equipos.

\subsection{La especie y fase de cultivo}

Uno de los requerimientos a la hora de seleccionar un equipo u otro de aireación, es tener en cuenta la especie a la cual va dirigido. Así mismo, dentro de la misma especie es correcto determinar si es necesario usar diferentes equipos de aireación a medida que avanzan las etapas de desarrollo. Es común para las fases iniciales de cultivo en tanques de menores dimensiones se busca mitigar las grandes turbulencias y permitir un mejor ambiente a los animales. Para eso, los sistemas de blower y difusores son los más utilizados (13). Al paso que el animal crece y si transfiere a los estanques de engorde (en el caso de cultivo multifásico), los animales ya están más adaptados y otros tipos de equipos pueden ser utilizados. Igualmente, es determinante aclarar que a medida que los animales crecen en tamaño, sus requerimientos por unidad de biomasa tienden a cambiar comparados a los animales menores (Tabla 2 y 3 ).

Tabla 2 Tasa de consumo de oxígeno $\left(\mathrm{mg} \mathrm{O}_{2} / \mathrm{L} / \mathrm{h}\right)$ de Litopenaeus vannamei calculada a partir de la tasa respiratoria individual $\left(\mathrm{mg} \mathrm{O}_{2} /\right.$ animal/h) y en función de las salinidades de 1,13,25 y $37 \mathrm{ppt}$, temperaturas de 20,25 y $30^{\circ} \mathrm{C}$, densidades de 10,40 y 120 individuos/m2 y peso húmedo de 5,1015 y 20 gr/camarón [43].

\begin{tabular}{cccccc}
\hline \multirow{2}{*}{ Sal. } & \multirow{2}{*}{ Temp. } & \multicolumn{4}{c}{$10 / \mathrm{m}^{2}$} \\
& & $5 \mathrm{gr}$ & $10 \mathrm{gr}$ & $15 \mathrm{gr}$ & $20 \mathrm{gr}$ \\
\hline \multirow{3}{*}{$37 \mathrm{ppm}$} & $20^{\circ} \mathrm{C}$ & 0,01 & 0,02 & 0,03 & 0,05 \\
& $25^{\circ} \mathrm{C}$ & 0,01 & 0,02 & 0,02 & 0,03 \\
& $30^{\circ} \mathrm{C}$ & 0,02 & 0,03 & 0,05 & 0,06 \\
& $20^{\circ} \mathrm{C}$ & 0,01 & 0,02 & 0,03 & 0,04 \\
$25 \mathrm{ppm}$ & $25^{\circ} \mathrm{C}$ & 0,01 & 0,03 & 0,04 & 0,05 \\
& $30^{\circ} \mathrm{C}$ & 0,02 & 0,03 & 0,05 & 0,06 \\
& $20^{\circ} \mathrm{C}$ & 0,01 & 0,02 & 0,04 & 0,05 \\
$13 \mathrm{ppm}$ & $25^{\circ} \mathrm{C}$ & 0,01 & 0,03 & 0,04 & 0,05 \\
& $30^{\circ} \mathrm{C}$ & 0,02 & 0,03 & 0,05 & 0,07 \\
& $20^{\circ} \mathrm{C}$ & 0,01 & 0,02 & 0,02 & 0,03 \\
$1 \mathrm{ppm}$ & $25^{\circ} \mathrm{C}$ & 0,01 & 0,03 & 0,04 & 0,06 \\
& $30^{\circ} \mathrm{C}$ & 0,02 & 0,04 & 0,10 & 0,14 \\
\cline { 2 - 6 } Sal. & Temp. & $5 \mathrm{gr}$ & $10 \mathrm{gr}$ & $15 \mathrm{gr}$ & $20 \mathrm{gr}$ \\
\hline $37 \mathrm{ppm}$ & $20^{\circ} \mathrm{C}$ & 0,03 & 0,08 & 0,13 & 0,18
\end{tabular}




\begin{tabular}{|c|c|c|c|c|c|}
\hline & $25^{\circ} \mathrm{C}$ & 0,05 & 0,07 & 0,09 & 0,11 \\
\hline & $30^{\circ} \mathrm{C}$ & 0,07 & 0,13 & 0,18 & 0,24 \\
\hline \multirow{3}{*}{25 ppm } & $20^{\circ} \mathrm{C}$ & 0,03 & 0,08 & 0,12 & 0,18 \\
\hline & $25^{\circ} \mathrm{C}$ & 0,05 & 0,10 & 0,15 & 0,20 \\
\hline & $30^{\circ} \mathrm{C}$ & 0,07 & 0,13 & 0,19 & 0,26 \\
\hline \multirow{3}{*}{13 ppm } & $20^{\circ} \mathrm{C}$ & 0,04 & 0,09 & 0,14 & 0,20 \\
\hline & $25^{\circ} \mathrm{C}$ & 0,05 & 0,10 & 0,15 & 0,21 \\
\hline & $30^{\circ} \mathrm{C}$ & 0,07 & 0,13 & 0,20 & 0,27 \\
\hline \multirow{3}{*}{1 ppm } & $20^{\circ} \mathrm{C}$ & 0,04 & 0,07 & 0,10 & 0,13 \\
\hline & $25^{\circ} \mathrm{C}$ & 0,05 & 0,10 & 0,16 & 0,22 \\
\hline & $30^{\circ} \mathrm{C}$ & 0,08 & 0,17 & 0,27 & 0,38 \\
\hline \multirow{2}{*}{ Sal. } & \multirow{2}{*}{ Temp. } & \multicolumn{4}{|c|}{$120 / \mathrm{m}^{2}$} \\
\hline & & $5 \mathrm{gr}$ & $10 \mathrm{gr}$ & $15 \mathrm{gr}$ & $20 \mathrm{gr}$ \\
\hline \multirow{3}{*}{37 ppm } & $20^{\circ} \mathrm{C}$ & 0,10 & 0,23 & 0,38 & 0,55 \\
\hline & $25^{\circ} \mathrm{C}$ & 0,15 & 0,22 & 0,28 & 0,34 \\
\hline & $30^{\circ} \mathrm{C}$ & 0,21 & 0,38 & 0,55 & 0,70 \\
\hline \multirow{3}{*}{25 ppm } & $20^{\circ} \mathrm{C}$ & 0,10 & 0,23 & 0,37 & 0,53 \\
\hline & $25^{\circ} \mathrm{C}$ & 0,15 & 0,30 & 0,45 & 0,61 \\
\hline & $30^{\circ} \mathrm{C}$ & 0,20 & 0,39 & 0,58 & 0,76 \\
\hline \multirow{3}{*}{13 ppm } & $20^{\circ} \mathrm{C}$ & 0,12 & 0,27 & 0,42 & 0,59 \\
\hline & $25^{\circ} \mathrm{C}$ & 0,15 & 0,30 & 0,46 & 0,62 \\
\hline & $30^{\circ} \mathrm{C}$ & 0,19 & 0,39 & 0,60 & 0,80 \\
\hline \multirow{3}{*}{1 ppm } & $20^{\circ} \mathrm{C}$ & 0,10 & 0,20 & 0,29 & 0,38 \\
\hline & $25^{\circ} \mathrm{C}$ & 0,14 & 0,31 & 0,48 & 0,65 \\
\hline & $30^{\circ} \mathrm{C}$ & 0,23 & 0,51 & 0,81 & 1,13 \\
\hline
\end{tabular}

Temp. Temperatura; Sal. Salinidad.

En la tabla 3 se presentan valores de consumo de oxígeno de referencia para diferentes especies que se usan o podrían ser cultivadas en este tipo de sistemas [23].

Tabla 3 Consumo de oxígeno de diferentes especies.

\begin{tabular}{|c|c|c|c|c|c|}
\hline Especie & $\begin{array}{c}\text { Tall } \\
\text { a } \\
(\mathrm{gr})\end{array}$ & $\begin{array}{c}\text { Temper } \\
\text { atura } \\
\left({ }^{\circ} \mathrm{C}\right)\end{array}$ & $\begin{array}{c}\text { Tasa de } \\
\text { alimentaci } \\
\text { ón }\end{array}$ & $\begin{array}{c}\text { Consu } \\
\text { mo de } \\
\text { oxígen } \\
\text { o (gr } \\
\mathrm{O}_{2} / \mathrm{kg} \\
\mathrm{pez} / \mathrm{hr})\end{array}$ & $\begin{array}{c}\text { Fuen } \\
\text { te }\end{array}$ \\
\hline \multirow[t]{2}{*}{$\begin{array}{l}\text { Salmo } \\
\text { gairdneri }\end{array}$} & 100 & 15 & - & 0,3 & [50] \\
\hline & $\begin{array}{c}100 \\
1\end{array}$ & 15 & $\begin{array}{c}\text { Niveles de } \\
\text { producció } \\
n\end{array}$ & 0,3 & [51] \\
\hline \multirow[t]{2}{*}{$\begin{array}{l}\text { Oncorhync } \\
\text { hus nerka }\end{array}$} & $\begin{array}{c}28 \\
1\end{array}$ & 15 & $\begin{array}{c}\text { Sin } \\
\text { alimento }\end{array}$ & 0,23 & [52] \\
\hline & $\begin{array}{c}28 \\
6\end{array}$ & 15 & $\begin{array}{c}3 \% \\
\text { Biomasa }\end{array}$ & 0,28 & \\
\hline \multirow[t]{2}{*}{$\begin{array}{l}\text { Ictalurus } \\
\text { puntatus }\end{array}$} & 100 & 30 & $\begin{array}{c}\text { Sin } \\
\text { alimento }\end{array}$ & 0,56 & \multirow{2}{*}{ [53] } \\
\hline & 100 & 30 & $\begin{array}{c}\text { A } \\
\text { Saciedad }\end{array}$ & 0,81 & \\
\hline \multirow[t]{3}{*}{$\begin{array}{l}\text { Cyprinus } \\
\text { carpio }\end{array}$} & 100 & 10 & Alimento & 0,17 & \multirow[t]{3}{*}{ [54] } \\
\hline & - & 20 & Alimento & 0,48 & \\
\hline & - & 25 & Alimento & 0,70 & \\
\hline \multirow{2}{*}{$\begin{array}{l}\text { Hypohthal } \\
\text { michthys } \\
\text { molitrix }\end{array}$} & 15 & 20 & - & 0,20 & [55] \\
\hline & 240 & 23 & Alimento & 0,25 & {$[56]$} \\
\hline \multirow[t]{3}{*}{$\begin{array}{l}\text { Orechromis } \\
\text { niloticus }\end{array}$} & 50 & 25 & $\begin{array}{c}\text { Sin } \\
\text { alimento }\end{array}$ & 0,16 & \multirow{3}{*}{ [57] } \\
\hline & 50 & 30 & $\begin{array}{c}\text { Sin } \\
\text { alimento }\end{array}$ & 0,24 & \\
\hline & 50 & 35 & $\begin{array}{c}\text { Sin } \\
\text { alimento }\end{array}$ & 0,40 & \\
\hline \multirow[t]{8}{*}{$\begin{array}{l}\text { Colossoma } \\
\text { macropom } \\
\text { un }\end{array}$} & 40 & 21 & Alimento & 184,55 & \multirow{8}{*}{ [59] } \\
\hline & 40 & 26 & Alimento & 241,47 & \\
\hline & 40 & 31 & Alimento & 281,92 & \\
\hline & 60 & 21 & Alimento & 162,60 & \\
\hline & 60 & 26 & Alimento & 227,99 & \\
\hline & 60 & 31 & Alimento & 254,37 & \\
\hline & 90 & 21 & Alimento & 96,07 & \\
\hline & 90 & 26 & Alimento & 142,06 & \\
\hline
\end{tabular}




\begin{tabular}{|c|c|c|c|c|c|}
\hline Especie & $\begin{array}{c}\text { Tall } \\
\text { a } \\
(g r)\end{array}$ & $\begin{array}{c}\text { Temper } \\
\text { atura } \\
\left({ }^{\circ} \mathrm{C}\right)\end{array}$ & $\begin{array}{c}\text { Tasa de } \\
\text { alimentaci } \\
\text { ón }\end{array}$ & $\begin{array}{c}\text { Consu } \\
\text { mo de } \\
\text { oxígen } \\
\mathrm{o}(\mathrm{gr} \\
\mathrm{O}_{2} / \mathrm{kg} \\
\mathrm{pez} / \mathrm{hr})\end{array}$ & $\begin{array}{c}\text { Fuen } \\
\text { te }\end{array}$ \\
\hline & 90 & 31 & Alimento & 208,43 & \\
\hline & 140 & 21 & Alimento & 66,73 & \\
\hline & 140 & 26 & Alimento & 115,17 & \\
\hline & 140 & 31 & Alimento & 160,33 & \\
\hline & 250 & 21 & Alimento & 53,98 & \\
\hline & 250 & 26 & Alimento & 82,82 & \\
\hline & 250 & 31 & Alimento & 114,17 & \\
\hline \multirow[t]{3}{*}{$\begin{array}{l}\text { Piaractus } \\
\text { brachypom } \\
\text { us }\end{array}$} & 30 & 18 & $\begin{array}{c}3 \% \\
\text { Biomasa }\end{array}$ & 127,3 & \multirow{3}{*}{ [41] } \\
\hline & 30 & 34 & $\begin{array}{c}3 \% \\
\text { Biomasa }\end{array}$ & 434,9 & \\
\hline & 480 & 34 & $\begin{array}{c}3 \% \\
\text { Biomasa }\end{array}$ & 127,36 & \\
\hline \multirow[t]{5}{*}{$\begin{array}{l}\text { Brycon } \\
\text { amazonicu } \\
s\end{array}$} & 100 & 28 & $\begin{array}{c}3 \% \\
\text { Biomasa }\end{array}$ & 288,5 & \multirow{5}{*}{ [60] } \\
\hline & 200 & 28 & $\begin{array}{c}3 \% \\
\text { Biomasa }\end{array}$ & 237,0 & \\
\hline & 400 & 28 & $\begin{array}{c}3 \% \\
\text { Biomasa }\end{array}$ & 208,6 & \\
\hline & 800 & 28 & $\begin{array}{c}3 \% \\
\text { Biomasa }\end{array}$ & 114,7 & \\
\hline & $\begin{array}{c}160 \\
0\end{array}$ & 28 & $\begin{array}{c}3 \% \\
\text { Biomasa }\end{array}$ & 124,6 & \\
\hline \multirow[t]{4}{*}{$\begin{array}{l}\text { Oreochromi } \\
\text { s sp }\end{array}$} & 25 & 18 & $\begin{array}{c}\% \\
\text { Biomasa }\end{array}$ & 176 & \multirow{4}{*}{ [42] } \\
\hline & 25 & 30 & $\begin{array}{c}3 \% \\
\text { Biomasa }\end{array}$ & 508 & \\
\hline & 800 & 18 & $\begin{array}{c}3 \% \\
\text { Biomasa }\end{array}$ & 96 & \\
\hline & 800 & 30 & $\begin{array}{c}3 \% \\
\text { Biomasa }\end{array}$ & 237 & \\
\hline
\end{tabular}

\section{FACTORES QUE DETERMINAN EL NUMERO DE AIREADORES}

Normalmente las bajas concentraciones de oxígeno disuelto se observan cuando los cultivos alcanzan elevadas biomasas de peces/camarones, además de elevadas cargas orgánicas suspendidas ("bioflocs") o decantadas (lodos o "sludge"), esencialmente al final de los cultivos. Bajas concentraciones de oxígeno disuelto (OD) pueden causar estrés y mortalidades (21). En este sentido, la aireación es un componente importante de gestión y control en los estanques que utilizan los sistemas BFT. Los niveles de OD deben proporcionar la oxidación de materia orgánica, garantizar los procesos de nitrificación, sobrevivencia de los animales y microrganismos (bioflocs).

Conociendo el sistema de aireación que se desea utilizar, es importante tener metodologías claras para establecer la cantidad de aireadores, con el fin de tener la mejor eficiencia posible dentro de los sistemas productivos. Según Avnimelech et al. (40), la cantidad de equipos de aeración suele ser determinada en campo por prueba y error, lo cual puede llevar a un sub o sobre dimensionamiento trayendo diversos prejuicios al cultivo.

Para determinar la cantidad de aireadores (o de OD necesario) en un sistema BFT se debe tomar en cuenta: 1) la demanda de $\mathrm{O}_{2}$ oriunda de la respiración de los peces o camarones, que por su vez dependerá de la especie a implementar, edad, carga y demás factores asociados a cada especie; y 2) la demanda propia de la respiración microbiana (39); Vinatea et al. (86). Esta última es muy variable y depende de diversos factores tales como concentración de sólidos presentes en la columna de agua (bioflocs), la cantidad (input) de nutrientes en el sistema, estatus trófico (autotrófico, heterotrófico o quimio autotrófica) (Vinatea et al. (77), eventuales niveles de sludge formado en los fondos, entre otros factores.

En relación con la carga máxima de sólidos suspendidos para camarones, (17) y (42) recomiendan valores de $<500 \mathrm{mg} / \mathrm{L}$ en sólidos suspendidos totales $y<15 \mathrm{ml} / \mathrm{L}$ en sólidos sedimentables (cono Imhoff), respectivamente. Aun en relación con la demanda de $\mathrm{O}_{2}$ oriunda de la respiración de los peces o camarones, se debe tomar en cuenta la carga máxima a ser alcanzada, una vez que esta incrementase con el tiempo en una misma infraestructura y la cantidad de OD incorporado debe ajustarse proporcionalmente. 
Es importante mencionar que, de manera muy general, animales de menor talla poseen un metabolismo más acelerado, y, en consecuencia, una mayor demanda de $\mathrm{O}_{2}$ por $\mathrm{kg}$ de biomasa. Es decir, $1 \mathrm{~kg}$ de alevines de tilapias consumen más oxígeno que $1 \mathrm{~kg}$ de tilapias adultas. Ebeling y Timmons (71) brindan una base de respiración de los peces con base en el alimento ingerido de 0,5 a $1 \mathrm{~kg}$ de oxígeno por cada kilogramo de alimento consumido en sistemas de recirculación. Avnimelech (31) menciona que el consumo de oxígeno varía entre 200 y $400 \mathrm{mg} \mathrm{O}_{2}^{-1} \mathrm{~kg}^{-1}$ peso vivo/hora para peces. Cabe resaltar que estos valores no toman en cuenta la respiración microbiana, que según Browdy et al. (66) en cultivos de camarones, puede representar más de $60 \%$ de total del consumo de oxígeno del estanque. Es importante destacar, que en sistemas BFT las biomasas máximas $\left(\mathrm{kg} / \mathrm{m}^{3}\right)$ utilizadas para peces son mayores que las utilizadas para camarón (2-5 $\mathrm{kg} / \mathrm{m}^{3-1}$ para camarón versus 7 hasta más de $20 \mathrm{~kg}$ $\mathrm{m}^{3-1}$ para peces, respectivamente). Sin embargo, como se mencionó anteriormente, la demanda de oxígenos por $\mathrm{kg}$ de biomasa es menor, resultando en mayor eficiencia comparado a los camarones.

Vinatea et al. (77) reportan para un cultivo de 610 camarones $\mathrm{m}^{2-1}$ en un raceways de $271 \mathrm{~m}^{2}$ una tasa respiratoria de los microrganismos en la columna de agua de 0,32 a 2,60 $\mathrm{mg} \mathrm{O}_{2} / \mathrm{L} / \mathrm{h}$. Estas diferencias están directamente relacionadas con la edad/peso de los animales (1,87 a 19,32 gr) y la cantidad de nutrientes (alimento balanceado) que entran a los sistemas. Además, también está directamente relacionado con la concentración de sólidos (generalmente monitoreados como SS, TSS y VSS) y la condición trófica presente en el momento, es decir, la relación entre las tasas de fotosíntesis y respiración microbiana. Además de las tasas de respiración microbiana, la respiración de los camarones varia 0,008 a $1,130 \mathrm{mg} \mathrm{O} / \mathrm{L} / \mathrm{h}$ de acuerdo con Vinatea et al. (77). Estas diferencias tan marcadas son debidas a las densidades de siembra $\left(10,40\right.$ y $\left.120 \mathrm{cam} / \mathrm{m}^{2}\right)$, salinidad $(1,13,25$ y $37 \mathrm{ppt})$ y temperatura del agua $\left(20,25\right.$ y $\left.30^{\circ} \mathrm{C}\right)$.

\section{PERSPECTIVAS FUTURAS}

En un mundo globalizado y de producción en masa a medida que la acuacultura intensiva en biofloc se va popularizando y ganando escala, es esperado que los costos de producción se vayan disminuyendo. Gran parte de estos costos son debido al uso continuo de los aparatos de aeración.

En este sentido, aun son necesarias más investigaciones para (i) desarrollar equipos más robustos y eficientes, incluso con evaluación de energías alternativas (paneles solares, molinos de viento, biodigestores, etc.); (ii) comparar en ambiente BFT los distintos equipos; (iii) conocer la demanda metabólica de OD de las distintas especies en sistemas BFT, incluyendo las especies nativas.

\section{AGRADECIMIENTOS}

A la Dirección General de Investigación Unillanos por parte de la financiación del proyecto. Código: C04-F01-008-2016. A la Doctora Katt Lapa por sus contribuciones al presente artículo. M. Emerenciano agradece a Fundação de Amparo à Pesquisa e Inovação do Estado de Santa Catarina FAPESC (PAP-FAPESC 2015TR453 e PAPFAPESC 2013TR3406).

\section{REFERENCIAS}

[1] Fao. Estado mundial de la pesca y la acuicultura - contribución a la seguridad alimentaria y la nutrición para todos. Roma2016

[2] Fao. El estado mundial de la pesca y la acuicultura 2018. Cumplir los objetivos de desarrollo sostenible. Roma. 2018

[3] Hardgreaves. Biofloc production system for aquaculture Southern Regional Aquaculture Center 4503. 2013

[4] Tidwell. Aquaculture production systems, WileyBlackwell.434. 2012

[5] Vinatea. Princípios químicos de qualidade de água em aquacultura: Uma revisão para peixes e camarões. Florianapolis2004

[6] Atencio-Garcia, Pertuz-Buelvas, Bru-Cordero and Ayazo-Genes. Curso teorico-práctico tecnológia en cultivo biofloc. CINPIC. Colombia, Universidad de Cordoba. 2013

[7] Seibert and Pinto. Challenges in shrimp aquaculture due to viral diseases: Distribution and biology of the five major penaeid viruses and interventions to avoid viral incidence and dispersion. Brazilian journal of microbiology : [publication of the Brazilian Society for Microbiology] 43(3). 857-864. 2012

[8] Zorriehzahra and Banaederakhshan. Early mortality syndrome (ems) as new emerging threat in 
shrimp industry. Advances in Animal and Veterinary Sciences 3. 64-72. 2015

[9] Martínez-Cordero and Sánchez. Enfermedades emergentes y cultivo de tilapia: Modelo conceptual de analisis de impactos en mexico del virus del lago de tilapia (tilv). LAQUA17, Mazatlan, Mexico, WAS.168. Year

[10] Stokstad. Down on the shrimp farm, American Association for the Advancement of Science. 2010

[11] Emerenciano, Martínez-Córdova, MartínezPorchas and Miranda-Baeza. Biofloc technology (bft): A tool for water quality management in aquaculture. Water quality, InTech. 2017

[12] Emerenciano, Gaxiola and Cuzon. Biofloc technology (bft): A review for aquaculture application and animal food industry. Biomass now - cultivation and utilization. 2013

[13] Avnimelech, Schryver, Emerenciano, Kuhn, Ray and Taw. Biofloc technology - a practical guide book. Baton Rouge, Louisiana, United States2015

[14] Schryver, Crab, Defoirt, Boon and W. The basics of biofloc technology: The added value for aquaculture. Aquaculture 277. 125-137. 2008

[15] Avnimelech. Carbon/nitrogen ratio as a control element in aquaculture systems. Aquaculture 176. 227-235. 1999

[16] Schenider, Sereti and Verret. Analysis of nutrient flows in integrated intensive aquaculture systems. Aquacult Eng 32(3-4). 379-401. 2005

[17] Samocha, Lopez, Jones, Jackson and Lawrence. Characterization of intake and effluent waters from intensive and semi-intensive shrimp farms in texas. Aquaculture research 35(4). 321-339. 2004

[18] Ebeling, Timmons and Bisogni. Engineering analysis of the stoichiometry of photoautotrophic, autotrophic, and heterotrophic removal of ammonianitrogen in aquaculture systems. Aquaculture 257(1). 346-358. 2006

[19] Van Wyk. Production of I. Vannamei in recirculating aquaculture systems: Management and design considerations. Proceedings of the 6th International Conference on Recirculation Aquaculture. Rakestraw, Douglas, Marsh et al. Roanoke, Virginia: 38-47. 2006

[20] Fao). Métodos sencillos para la acuicultura. Coleccion FAO Capacitación Retrieved Marzo, 2015, from ftp://ftp.fao.org/fi/CDrom/FAO_training/FAO_training /general/x6709s/Index.htm.Pages. 2003

[21] Boyd. Pond water aeration systems. Aquacult Eng 18. 9-40. 1998
[22] Rogers. Varied aerator designs provide oxygenation, mixing in biofloc systems. Global Aquaculture Advocate 13(5). 50,51. 2010

[23] Vinatea. Vinatea, I. Princípios químicos de qualidade da água em aqüicultura, segunda edição revisada e ampliada. Florianópolis, Editorial UFSC2004

[24] Vinatea and Carvalho. Influence of water salinity on the sotr of paddlewheel and propelleraspirator-pump aerators, its relation to the number of aerators per hectare and electricity costs. Aquacult Eng 37. 73-78. 2007

[25] Tucker. Pond aeration. Southern Regional Aquaculture Center 3700. 8. 2005

[26] Cancino. Design of high efficiency surface aerators: Part 2. Rating of surface aerator rotors. . Aquacult Eng 31(1-2). 99-115. 2004

[27] Monroy-Dosta, Lara-Andrade, Castro-Mejia, Castro-Mejia and Coelho-Emereciano. Composición y abundancia de comunidades microbianas asociadas al biofloc en un cultivo de tilapia. Revista de Biologia Marina y Oceanografía 48(3). 511-520. 2013

[28] Krummenauer, Poersch and Fóes. Sistemas de injetores de ar nos cultivos superintensivos em meio aos bioflocos. Panorama da Aquicultura. 25-31. 2016

[29] Lara, Krummenauer, Abreu, Poersch and Wasielesky. The use of different aerators on litopenaeus vannamei biofloc culture system: Effects on water quality, shrimp growth and biofloc composition. Aquaculture International 25(1). 147162. 2017

[30] Creswell and Hoff. Aquaculture desk reference, Florida Aqua Farms Incorporated2000

[31] Pöpel and Wagner. Prediction of oxygen transfer from simple measurements of bubble characteristics. Wat. Sci. Tech. 23. 1941-1950. 1991 [32] Motarjemi and Jameson. Mass tranfer from very small bubbles - the optimun bubble size for aeration. Chem Eng-New York 33. 1415-1423. 1978 [33] Hauser, Oberender, Richter and Muller. Interfacial turbulence enhances oxygen transport into shallow liquid layers. Physica D 205. 170-180. 2005

[34] Schierholza, Gulliverb, Wilhelmsc and Hennemand. Gas transfer from air diffusers. Water Res 40. 1018-1026. 2006

[35] Lima-Neto. Modeling the liquid volume flux in bubbly jets using a simple integral approach. . J Hydraul Eng-Asce 138(2). 210-215. 2012; LimaNeto. Turbulência induzida por jatos bifásicos do tipo 
gás-líquido em tanques de aeração. Engenharia Sanitária e Ambiental 15(1). 75-82. 2010

[36] Burford. Microbial communities affect water quality shrimp performance at belize aquaculture. Global Aquaculture Alliance. 2003

[37] Lawson and Merry. Procedures for evaluating low-power surface aerators under field conditions. Techniques for Modern Aquaculture. ASAE. Michigan, USA: 511.1993

[38] Liang, Luo, Tan, Ma, Zhang and Li. Efficiency of biofloc technology in suspended growth reactors treating aquacultural solid under intermittent aeration. Aquacult Eng 59. 41-47. 2014

[39] Vinatea. Oxygen consumption of litopenaeus vannamei juveniles in heterotrophic medium with zero water exchange. Pesq. Agropec. Bras. 44. 534538. 2009

[40] Vinatea, Muedas and Arantes. The impact of oxygen consumption by the shrimp litopenaeus vannamei according to body weight, temperature, salinity and stocking density on pond aeration: A simulation. Acta Scientiarum. Biological Sciences 33(2). 2011

[41] Vinatea, Gálvez, Browdy, Stokes, Venero, Haveman, Lewis, Lawson, Shuler and Leffler. Photosynthesis, water respiration and growth performance of litopenaeus vannamei in a superintensive raceway culture with zero water exchange: Interaction of water quality variables. Aquacultural Engineering 42(1). 17-24. 2010

[42] Taw. Commercial shrimp (litopenaeus vannamei) farming using biofloc system. 2010 [43] Ebeling and Timmons. Recirculation aquaculture systems. Aquaculture production systems. Tidwell. New Delhi, India, Wiley-Blackwell: 245-277. 2012

[44] Avnimelech. Biofloc technology - a practical guide book, World Aquaculture Society2009

[45] Browdy, Ray, Leffler and Avnimelech. Bioflocbased aquaculture system. Aquaculture production systems Tidwell. New Delhi, India, Wiley-Blackwell. 1. 2012 